\title{
Comparison between audio-only and audiovisual biofeedback for regulating patients' respiration during four-dimensional radiotherapy
}

\author{
Jesang Yu, MD, Ji Hoon Choi, MD, Sun Young Ma, MD, PhD, \\ Tae Sig Jeung, MD, PhD, Sangwook Lim, PhD \\ Department of Radiation Oncology, Kosin University Gospel Hospital, Kosin University College of Medicine, Busan, Korea
}

\begin{abstract}
Purpose: To compare audio-only biofeedback to conventional audiovisual biofeedback for regulating patients' respiration during four-dimensional radiotherapy, limiting damage to healthy surrounding tissues caused by organ movement.

Materials and Methods: Six healthy volunteers were assisted by audiovisual or audio-only biofeedback systems to regulate their respirations. Volunteers breathed through a mask developed for this study by following computer-generated guiding curves displayed on a screen, combined with instructional sounds. They then performed breathing following instructional sounds only. The guiding signals and the volunteers' respiratory signals were logged at 20 samples per second.

Results: The standard deviations between the guiding and respiratory curves for the audiovisual and audio-only biofeedback systems were $21.55 \%$ and $23.19 \%$, respectively; the average correlation coefficients were 0.9778 and 0.9756 , respectively. The regularities between audiovisual and audio-only biofeedback for six volunteers' respirations were same statistically from the paired t-test.

Conclusion: The difference between the audiovisual and audio-only biofeedback methods was not significant. Audio-only biofeedback has many advantages, as patients do not require a mask and can quickly adapt to this method in the clinic.
\end{abstract}

Keywords: Audio feedback, Respiratory regulation, Guided breath, Four dimensional radiotherapy

\section{Introduction}

Normal tissue damage during lung cancer radiotherapy is generally due to large treatment margins. Organ motion due to respiration requires that large margins be included in the planning target volume for adequate dose coverage in the chest and abdomen [1]. There are a few techniques available to minimize respiratory induced organ motion, such as active breathing control that holds patients' breaths spontaneously $[2-6]$, respiration gated radiation therapy $[7,8]$, and adaptation of radiation fields to a moving target by continuously utilizing information on the location of the internal target by direct or indirect monitoring [9-12]. To administer respiration adaptive radiation therapy effectively, it is essential to regulate patients' respiration, and many researchers have explored this concept [13-16]. We previously explored regulating respiration using audiovisual biofeedback, which regenerated patients' actual breath signals iteratively [17]. The audiovisual biofeedback was effective for regulating patients' respiration; however, a screen had to be installed and the patient had to wear the

Received 24 August 2015, Revised 7 September 2015, Accepted 11 September 2015.

Correspondence: Sangwook Lim, PhD, Department of Radiation Oncology, Kosin University Gospel Hospital, Kosin University College of Medicine, 262 Gamcheon-ro, Seo-gu, Busan 49267, Korea. Tel: +82-51-990-6393, Fax: +82-2-6280-6247, E-mail: medicalphysics@hotmail.com

(c) This is an Open Access article distributed under the terms of the Creative Commons Attribution Non-Commercial License (http://creativecommons.org/ licenses/by-nc/4.0/) which permits unrestricted non-commercial use, distribution, and reproduction in any medium, provided the original work is properly cited.

www.e-roj.org 
goggle for obtaining visual feedback. Adapting the patients to the audiovisual biofeedback devices to the patients needed at least 10 minutes in clinics. Some busy departments may have difficulties in setting up such devices.

Conversely, the audio-only feedback method does not require the installation of screen or wearing the goggle for video guidance in the treatment room; patients merely listen to a sound emitted from a speaker fixed to the wall or ceiling.

The purpose of this study was to compare the effectiveness of audiovisual versus audio-only biofeedback, and to see whether audio-only biofeedback is a practical alternative for regulating patients' respiration during four-dimensional radiation therapy.

\section{Materials and Methods}

\section{Respiration monitoring system}

The respiration monitoring mask (ReMM) was previously developed with a chromel-alumel (K-type) thermocouple to measure respiration while indirectly monitoring tumor motion $[18,19]$. The thermocouple measures temperature changes at the center of the air stream from the nose and mouth in a commercial dustproof mask (N7000; Woori Tech Co., Seongnam, Korea) (Fig. 1). The cool air from outside the mask flows into the mask when the patient inhales, and the mask is filled with warm air from a patient's lung when he/she exhales. In this manner, the thermocouple measures the change of temperature in the mask as the patient breathes. The end of the thermocouple was connected to the operational amplifier chip (AD597AH; Analog Devices, Norwood, MA, USA). The thermocouple signals were digitized in real time with a data acquisition card (DA0 6024E; National Instruments, Austin TX, USA). Continuous temperature readings corresponding to the respiration were analyzed and stored at 100 samples per second. The program for acquiring and analyzing respiratory signals was built using LabVIEW 7.0 (National Instruments).

A system that regulates a subject's respiration consists of a ReMM, a thermocouple module, the analog-to-digital converter card, one screen each for the operator and the patient (although only one was used in this study for the volunteers), a speaker, and a computer.

\section{Patients' respiration regulating system}

In order to enable the patients to breathe regularly, we developed the audiovisual biofeedback system using the ReMM in a previous study [17]. The setup of this system is the same as shown in Fig. 1. The program was optimized to allow audiovisual feedback. Two curves are displayed on the screen: one indicates the patient's current respiration pattern and the other is a guiding signal, which represents a single respiration cycle that was acquired by monitoring each volunteer's regular respiration with the ReMM and was then continuously reproduced (Fig. 2). The guiding sound was a beep; alternatively, Korean and English language instruction voices were available (Fig. 3). Audio biofeedback was for auxiliary guidance in case the patient looked away from the screen displaying the guiding curve for any reason.

\section{Audiovisual versus audio-only biofeedback}

All volunteers were instructed to breathe following their own guiding curve, and to control their breath by watching the screen and listening to the audio instruction. Six healthy

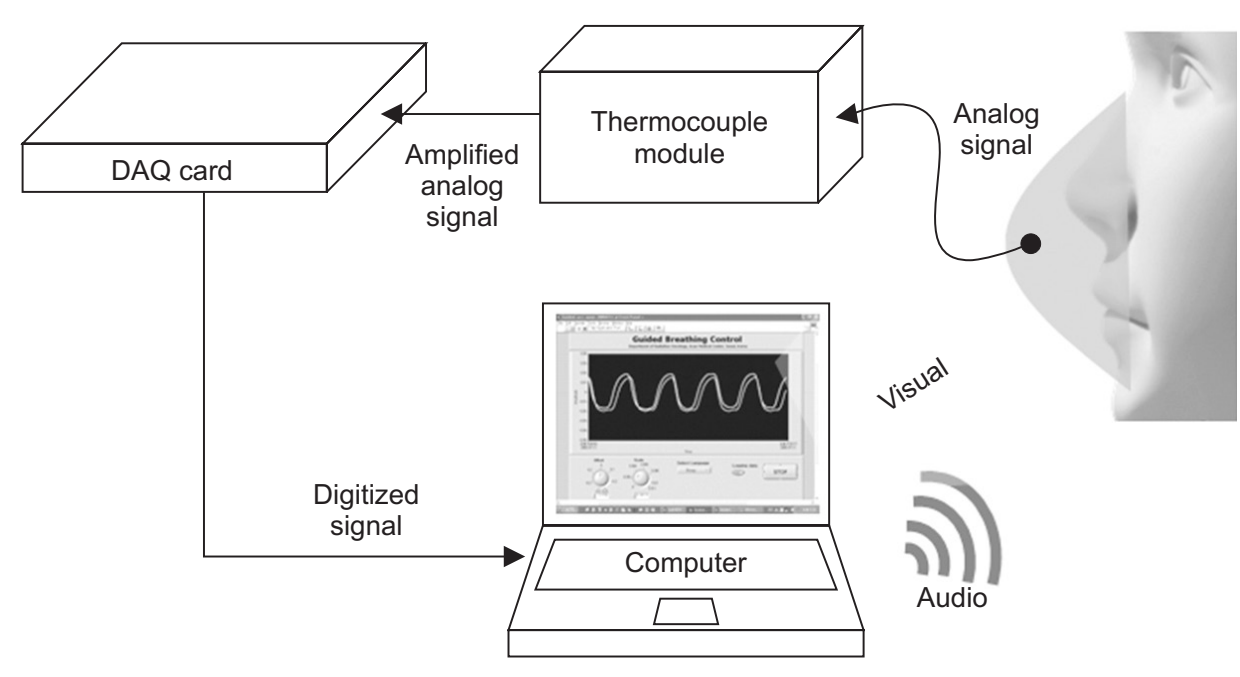

Fig. 1. Schematic diagram of the Audiovisual Guided Breath System. The respiratory signal from the respiration monitoring mask is amplified by the thermocouple module and digitized by the analogto-digital converter. The computer generates the guiding signal and acquires the respiratory signal. DAO, data acquisition. 
A

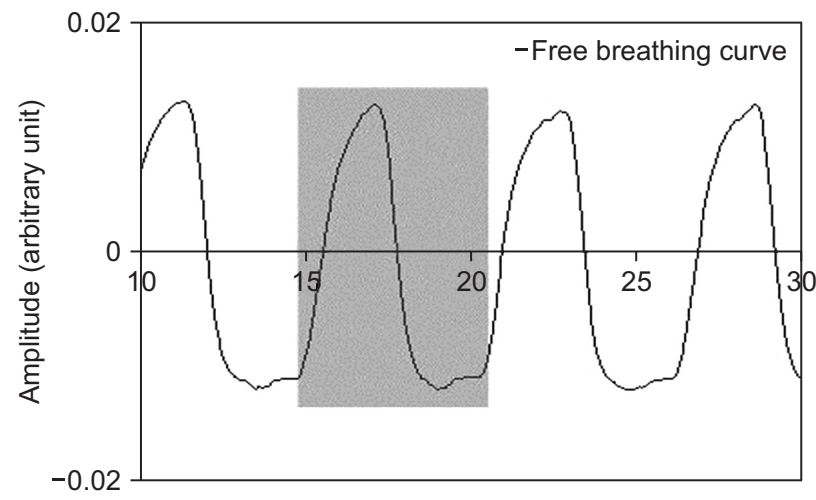

Elapsed time (s)
B

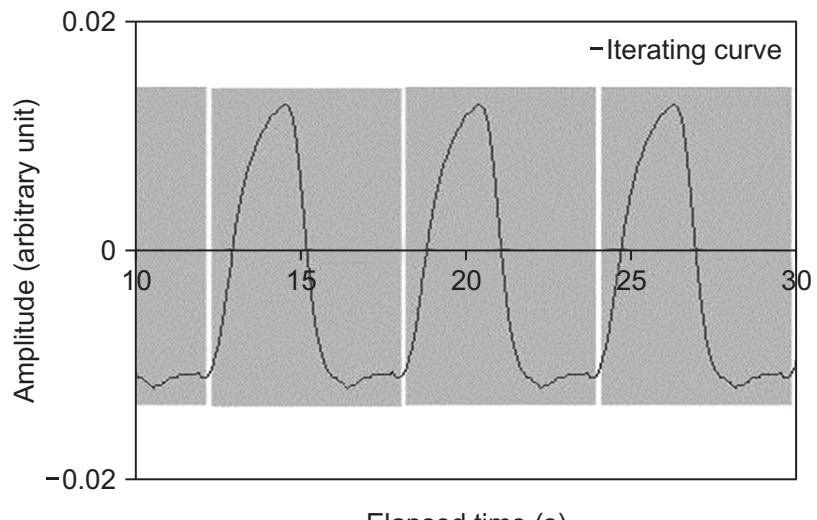

Elapsed time (s)

Fig. 2. Generating the guiding curve. (A) Free respiration: a single curve cycle is extracted from the actual respiration during normal respiration for each volunteer. (B) Guiding curve: the single extracted curve from panel A was then iterated.
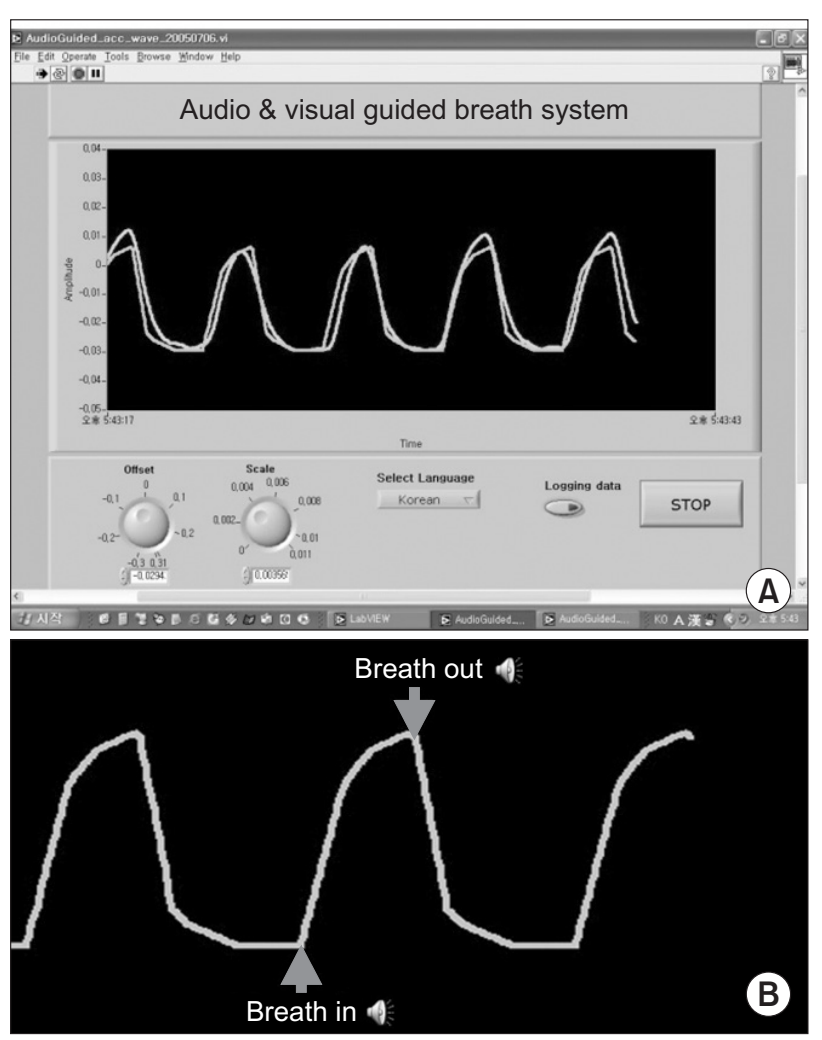

Fig. 3. (A) Audiovisual assistance: the gray curve represents the guiding curve while the green curve represents the respiratory curve. (B) Audio assistance: this was programmed to sound out the words "breath out" when the curve on the peak, and "breath in" when on the trough. The sounds were based on the guiding curve. A preferred language (Korean or English) could be selected; otherwise, a beep would sound instead. volunteers are enrolled to test this system. After a 5-minute instruction session, volunteers breathed through the ReMM by following the guiding curves and sounds simultaneously; in the subsequent session, they followed only the sound guidance based on the guiding curve. The guiding signal and the volunteers' respiration signals were logged at 20 samples per second. Volunteers' respirations with both biofeedback assistances were logged using the ReMM for 60 seconds, respectively. Then we calculated standard deviation of amplitude in percent (amplitude of guiding curve is 100\%) and correlation coefficient for volunteers' respiration signal comparing to its guiding curve. The correlation coefficient takes into account shape differences of the curves and the standard deviation is for evaluating the difference of amplitudes.

\section{Results}

The system responded dynamically to the volunteers' respiration signals, which were displayed directly on the screen. The delay time was of the order of a few milliseconds, which is negligible in clinics. Volunteers followed the guided curves effortlessly, as the system continuously generates respiration cycle representative of their actual respiration patterns. The volunteers were more comfortable with the audio-only biofeedback than with the audiovisual biofeedback procedure because they did not require the screen and goggle.

Table 1 shows the correlation coefficient and the standard deviation of amplitudes between the reference signal (the guidance curves) and the volunteers' respiration curves for both audiovisual and audio-only biofeedback. The average 
correlation coefficients between the guidance and the respiration curves for volunteers assisted by audiovisual and audio-only biofeedback systems were estimated as 0.9778 and 0.9756 , respectively. The standard deviation between the

Table 1. Relationship between the guiding curve extracted from the actual respirations of six volunteers and the respiration signal: audiovisual feedback was slightly superior to audio-only biofeedback

\begin{tabular}{lccccc}
\hline \multirow{2}{*}{ Volunteer } & \multicolumn{2}{c}{ Standard deviation (\%) } & & \multicolumn{2}{c}{ Correlation coefficient } \\
\cline { 2 - 3 } \cline { 5 - 6 } & Audiovisual & Audio & & Audiovisual & Audio \\
\hline 1 & 18.19 & 24.70 & & 0.9863 & 0.9707 \\
2 & 20.32 & 23.94 & & 0.9824 & 0.9832 \\
3 & 22.07 & 25.18 & & 0.9767 & 0.9684 \\
4 & 20.71 & - & & 0.9784 & - \\
5 & 20.28 & 21.65 & & 0.9795 & 0.9763 \\
6 & 27.70 & 20.49 & & 0.9635 & 0.9795 \\
Average & 21.55 & 23.19 & & 0.9778 & 0.9756 \\
p-value & \multicolumn{3}{c}{0.335} & & \multicolumn{3}{c}{0.837} \\
\hline
\end{tabular}

guiding curves and the respiration curves were $21.55 \%$ and 23.19\% for the audiovisual and the audio-only biofeedback, respectively. The significance levels of differences between guiding and respiration curves for audiovisual and audioonly biofeedback were evaluated. The regularities of both biofeedback were not different statistically with $p$-values of 0.335 and 0.837 for standard deviation and correlation coefficient, respectively, at the 5\% significant level from the paired t-test. Fig. 4 shows the first volunteer's respiration curves. Fig. $4 \mathrm{~A}$ and $\mathrm{B}$ show the respiration data obtained by audio-only biofeedback assistance, whereas Fig. 4C and D show respiration data obtained by audiovisual biofeedback assistance. The audiovisual biofeedback was slightly superior to the audio-only biofeedback according to our data.

\section{Discussion and Conclusion}

Monitoring fluoroscopic images is the most direct method to measure the respiratory induced organ motion. However
A

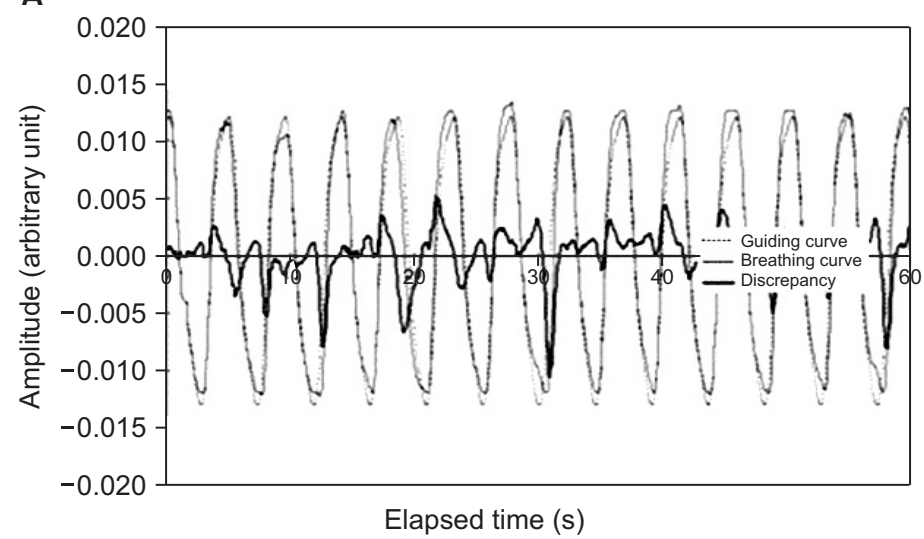

C

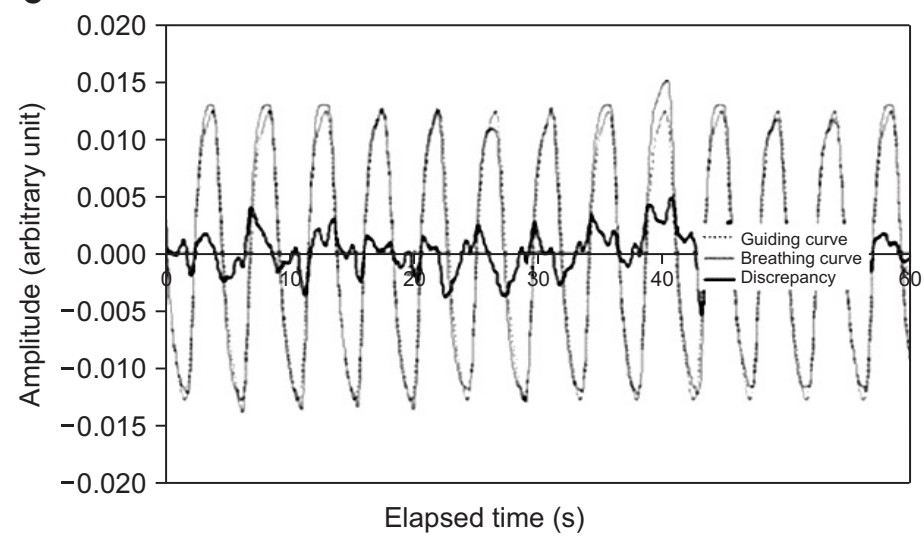

B

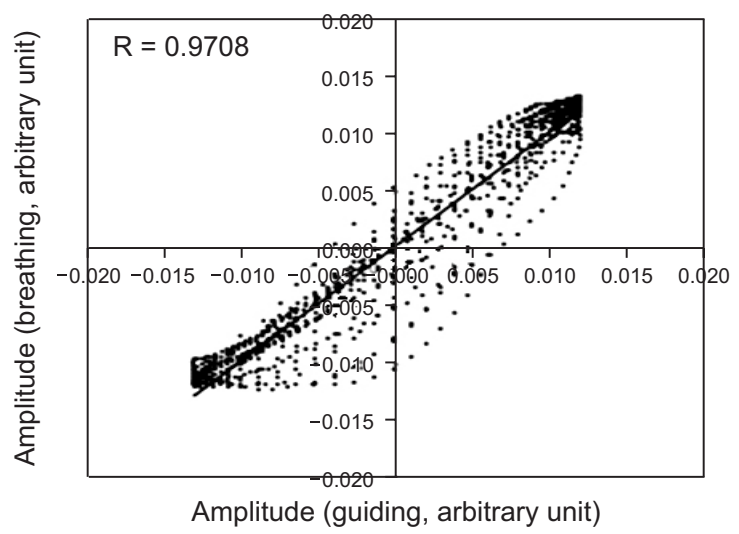

D

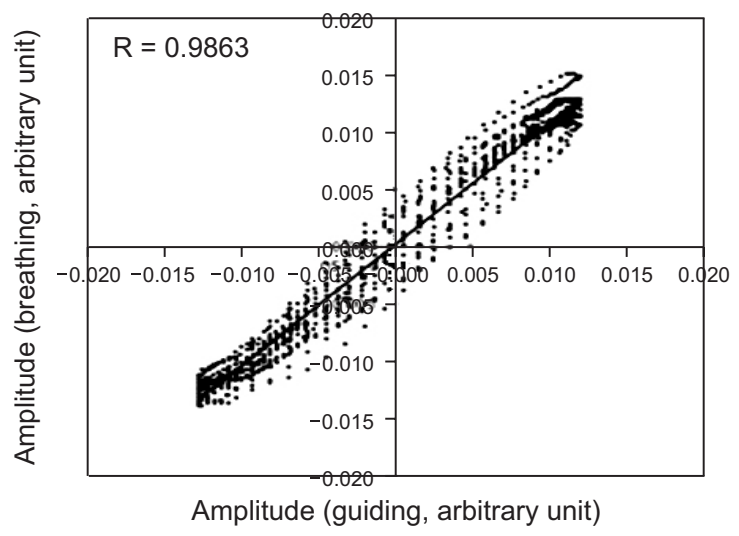

Fig. 4. Comparison between guiding curves and respiration curves for volunteer 1 (A) the discrepancy between the audio-only guiding curve and respiration curve. (B) The correlation between the two sets of curves in panel A. (C) The discrepancy between the audiovisual guiding curve and respiration curve. (D) The correlation between the two curves in panel C. 
unnecessary radiation doses can be delivered to patients $[9,20-$ 22]. The Real-time Position Management (RPM) which detect skin motion using infrared reflectors is a commercialized tool for monitoring organ motion indirectly. A spirometer can also be used to measure respiration; however, there is a problem of baseline-level drift of respiratory signal. These indirect methods require correlation with the target motion. Kubo and Hill [7] showed that the temperature sensor is reproducible and accurate. The temperature sensor has many advantages for monitoring target motion for 4D radiotherapy. The ReMM made with thermocouple showed a strong correlation with respiratory induced organ motion in the previous study, indicating that organ motion could be reliably determined from the information obtained via ReMM [19]. The respiration regulating system developed in the previous study used the audiovisual biofeedback method [17]. Patients were comfortable with the guiding curves, since the system continuously generates a respiration cycle based on their own.

However, audio-only biofeedback has many advantages over audiovisual biofeedback, particularly in overburdened clinics. Patients do not require the screen and goggle and can adapt quickly to the system. In this study, we aimed to show that audio-only biofeedback is also practical, especially as the difference between audio-only versus audiovisual biofeedback was not significant. The limitation of this study is the number of volunteers and the fourth volunteer did not perform audioonly biofeedback assistance. The sequence of measurements of audiovisual and audio-only biofeedback could result in bias in overestimating regularity of audio-only biofeedback assistance. In the future we need to evaluate this study with enough volunteers and alternative sequences. The respiration regulation is not mandatory but it has been reported that the more regular respiration, the more effective $4 D$ radiotherapy such as gated or breath hold radiotherapy [13-17]. This study showed that audio-only biofeedback was simple and practical method to regulate respiration.

\section{Conflict of Interest}

No potential conflict of interest relevant to this article was reported.

\section{Acknowledgments}

This research was supported by Basic Science Research Program through the National Research Foundation of Korea funded by the Ministry of Education, Science and Technology
(2010-0013701 and 2013R1A1A2012013).

\section{References}

1. Keall PJ, Mageras GS, Balter JM, et al. The management of respiratory motion in radiation oncology report of AAPM Task Group 76. Med Phys 2006;33:3874-900.

2. Wilson EM, Williams FJ, Lyn BE, Wong JW, Aird EG. Validation of active breathing control in patients with non-small-cell lung cancer to be treated with CHARTWEL. Int J Radiat Oncol Biol Phys 2003;57:864-74.

3. Stromberg JS, Sharpe MB, Kim LH, et al. Active breathing control $(A B C)$ for Hodgkin's disease: reduction in normal tissue irradiation with deep inspiration and implications for treatment. Int J Radiat Oncol Biol Phys 2000;48:797-806.

4. Mah D, Hanley J, Rosenzweig KE, et al. Technical aspects of the deep inspiration breath-hold technique in the treatment of thoracic cancer. Int J Radiat Oncol Biol Phys 2000;48:117585.

5. Rosenzweig KE, Hanley J, Mah D, et al. The deep inspiration breath-hold technique in the treatment of inoperable nonsmall-cell lung cancer. Int J Radiat Oncol Biol Phys 2000;48: 81-7.

6. Barnes EA, Murray BR, Robinson DM, Underwood $\sqcup$, Hanson J, Roa WH. Dosimetric evaluation of lung tumor immobilization using breath hold at deep inspiration. Int J Radiat Oncol Biol Phys 2001;50:1091-8.

7. Kubo HD, Hill BC. Respiration gated radiotherapy treatment: a technical study. Phys Med Biol 1996;41:83-91.

8. Ramsey CR, Scaperoth D, Arwood D, Oliver AL. Clinical efficacy of respiratory gated conformal radiation therapy. Med Dosim 1999;24:115-9.

9. Shimizu $S$, Shirato $H$, Ogura $S$, et al. Detection of lung tumor movement in real-time tumor-tracking radiotherapy. Int J Radiat Oncol Biol Phys 2001;51:304-10.

10. Keall PJ, Kini VR, Vedam SS, Mohan R. Motion adaptive x-ray therapy: a feasibility study. Phys Med Biol 2001;46:1-10.

11. Schweikard A, Shiomi $H$, Adler J. Respiration tracking in radiosurgery. Med Phys 2004;31:2738-41.

12. D'Souza WD, Naqvi SA, Yu CX. Real-time intra-fraction-motion tracking using the treatment couch: a feasibility study. Phys Med Biol 2005;50:4021-33.

13. Suh Y, Yi B, Ahn S, et al. Aperture maneuver with compelled breath (AMC) for moving tumors: a feasibility study with a moving phantom. Med Phys 2004;31:760-6.

14. He $P$, Li $Q$, Liu $X$, et al. Respiratory motion management using audio-visual biofeedback for respiratory-gated radiotherapy of synchrotron-based pulsed heavy-ion beam delivery. Med Phys 2014;41:111708.

15. Pollock S, Lee D, Keall P, Kim T. Audiovisual biofeedback 
improves motion prediction accuracy. Med Phys 2013;40: 041705.

16. Masselli GM, Silvestri S, Ramella S, Trodella L. Design and evaluation of a methodology to perform personalized visual biofeedback for reducing respiratory amplitude in radiation treatment. Med Phys 2009;36:1467-72.

17. Lim S, Park SH, Ahn SD, et al. Guiding curve based on the normal breathing as monitored by thermocouple for regular breathing. Med Phys 2007;34:4514-8.

18. Lim S, Ahn SD, Park SH, et al. Study of respiration simulating phantom using thermocouple-based respiration monitoring mask. J Korean Soc Ther Radiol Oncol 2005;23:217-22.

19. Lim S, Jeung TS, Park SH, Ahn SD, Lee S, Kim CY. Determination of target motion by using a respiration monitoring mask for 4-D radiotherapy. J Korean Phys Soc 2008;53:845-50.

20. Ahn YC, Shimizu S, Shirato $H$, et al. Application of real-time tumor-tracking and gated radiotherapy system for unresectable pancreatic cancer. Yonsei Med J 2004:45:584-90.

21. Shimizu S, Shirato $H$, Kagei $K$, et al. Impact of respiratory movement on the computed tomographic images of small lung tumors in three-dimensional (3D) radiotherapy. Int J Radiat Oncol Biol Phys 2000;46:1127-33.

22. Balter JM, Ten Haken RK, Lawrence TS, Lam KL, Robertson JM. Uncertainties in CT-based radiation therapy treatment planning associated with patient breathing. Int J Radiat Oncol Biol Phys 1996;36:167-74. 